\title{
Scaling of cellular proteome with ploidy
}

\author{
Galal Yahya ${ }^{1,2}$, Paul Menges ${ }^{1}$, Devi Anggraini Ngandiri ${ }^{1}$, Daniel Schulz ${ }^{3}$, Andreas Wallek ${ }^{4}$, Nils Kulak ${ }^{4}$, \\ Matthias Mann ${ }^{4}$, Patrick Cramer $^{5}$, Van Savage ${ }^{6}$, Markus Raeschle ${ }^{1}$, Zuzana Storchova $^{1^{*}}$
}

\begin{abstract} remodeling in response to increased ploidy.

${ }^{1}$ Dept. of Molecular Genetics, TU Kaiserslautern, Paul-Ehrlich-Strasse 24, 67663 Kaiserslautern, Germany.

${ }^{2}$ Department of Microbiology and Immunology, School of Pharmacy, Zagazig University, Egypt.

${ }^{3}$ Institute of Molecular Biology, University of Zurich, Switzerland

${ }^{4}$ Max Planck Institute of Biochemistry, 82152 Martinsried, Germany

${ }^{5}$ Max Planck Institue of Biophysical Chemistry, Goettingen, Germany

${ }^{6}$ Department of Biomathematics, University of California at Los Angeles, Los Angeles, CA 90095, United States

*Correspondence to: Zuzana Storchova, storchova@bio.uni-kl.de
\end{abstract}

Ploidy changes are frequent in nature and contribute to evolution, functional specialization and tumorigenesis $(1,2)$. Analysis of model organisms of different ploidies revealed that increased ploidy leads to an increase in cell and nuclear volume, reduced proliferation (2-4), metabolic changes (5), lower fitness $(6,7)$, and increased genomic instability $(8,9)$, but the underlying mechanisms remain poorly understood. To investigate how the gene expression changes with cellular ploidy, we analyzed isogenic series of budding yeasts from $1 \mathrm{~N}$ to $4 \mathrm{~N}$. We show that mRNA and protein abundance scales allometrically with ploidy, with tetraploid cells showing only threefold increase in proteins compared to haploids. This ploidy-specific scaling occurs via decreased rRNA and ribosomal protein abundance and reduced translation. We demonstrate that the Tor 1 activity is reduced with increasing ploidy, which leads to rRNA gene repression via a novel Tor1-Sch9-Tup1 signaling pathway. mTORC1 and S6K activity are also reduced in human tetraploid cells and the concomitant increase of the Tup1 homolog Tle1 downregulates the rDNA transcription. Our results revealed a novel conserved mTORC1-S6K-Tup1/Tle1 pathway that ensures proteome

The majority of eukaryotic organisms are diploid ( $2 \mathrm{~N}$, with two sets of chromosome) or haploid $(1 \mathrm{~N})$, but polyploid cells $(>2 \mathrm{~N})$ are common in nature. Polyploidy is found throughout the eukaryotic kingdom and plays an important role in speciation, especially in plants. Here, polyploidy increases adaptive potential, bringing short-term success but also disadvantages, reflected in the fact that the number of established whole-genome duplications (WGDs) is low $(10,11)$. Additionally, polyploidy plays an important role in differentiation of multicellular organisms, where it arises from developmentally tightly controlled polyploidization or in response to stress conditions in specialized organs and tissues (5). Polyploidy can also occur from an error. Unscheduled polyploidy is common in human cancers, and an estimated $37 \%$ of all cancers underwent a WGD event at some point during their progression (12); the incidence of WGD is even higher in metastasis (13). In addition, tetraploidy promotes tumorigenesis in cancer model systems (14). WGD generally impairs genome stability (9,15-18), but also increases cell evolvability and adaptability (19) and it is therefore considered an important driving force in evolution and tumorigenesis $(1,20)$. Despite the frequent occurrence of polyploidy and its importance in evolution and pathology, the effects of WGD on cellular physiology are only partially understood.

Budding yeasts Saccharomyces cerevisiae of different ploidy can be constructed and serve as an excellent model to study the consequences of WGD. An apparent result of increased ploidy is the linear increase in cell and nuclear volume, accompanied by a nonlinear (1.58-fold) scaling of two-dimensional structures such as membranes, and a 1.26-fold increase in linear structures $(2,9)$.
Polyploid budding yeasts grow at slightly reduced rates compared with diploids (2-4), reaching a larger volume in a similar time span. Increased ploidy in yeast leads to aberrant cell cycle regulation and response to nutrition $(2,21)$, lower fitness $(6,7)$, impaired genome stability $(8,9)$ and prominent evolvability (19). The inherent genome instability of tetraploid yeasts leads to a ploidy reduction to diploidy during in vitro evolution, likely due to the loss of individual chromosomes $(3,19)$. Analyses of the available transcriptomes show that the expression of only a handful of genes changes disproportionately in response to ploidy, suggesting that polyploidization largely tends to maintain the balance of gene products $(2,9,22)$. The few genes with altered mRNA abundance encode membrane and cell wall proteins, likely reflecting an adaptation to the lower surface-to-volume ratio in larger polyploid cells $(2,22)$. The minimal changes observed in transcriptome raised the question of whether ploidy-dependent regulation occurs post-transcriptionally. Therefore, we set out to analyze proteome changes in response to altered ploidy.

We generated a series of isogenic haploid to tetraploid strains derived from the BY4748 strain background, all with the mating type MATa to eliminate the effects of the pheromone pathway (Extended Data 1A, Supplementary Table 1). Ploidy was confirmed by flow cytometry (Extended Data 1B). As expected, the volume of budding yeast cells increased linearly with ploidy $(2,9)$, with median volumes of $48.0 \mathrm{fl}$ for $1 \mathrm{~N}, 82.9 \mathrm{fl}$ for $2 \mathrm{~N}, 146.6 \mathrm{fl}$ for $3 \mathrm{~N}$ and $181.7 \mathrm{fl}$ for $4 \mathrm{~N}$ (Extended Data 1C, D). 
A

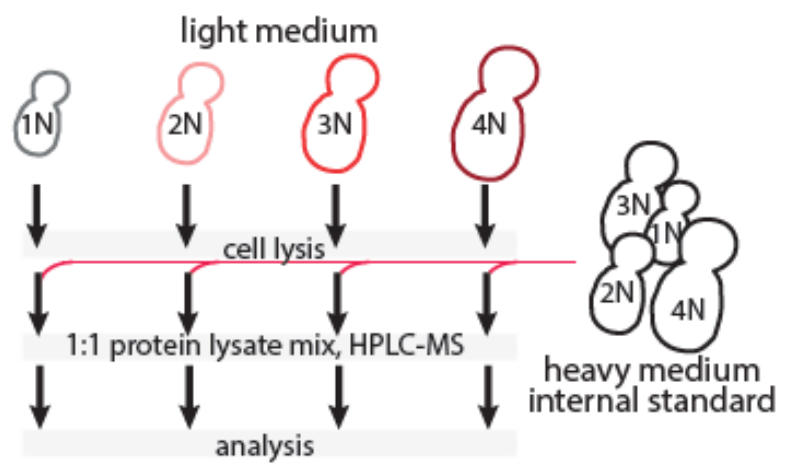

C

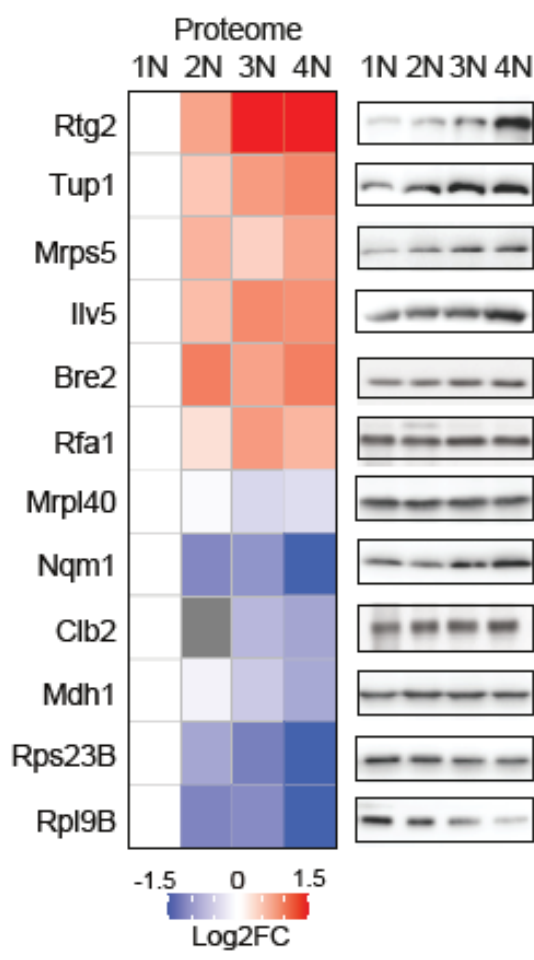

Immunoblotting

$1 \mathrm{~N} 2 \mathrm{~N} 3 \mathrm{~N} 4 \mathrm{~N}$

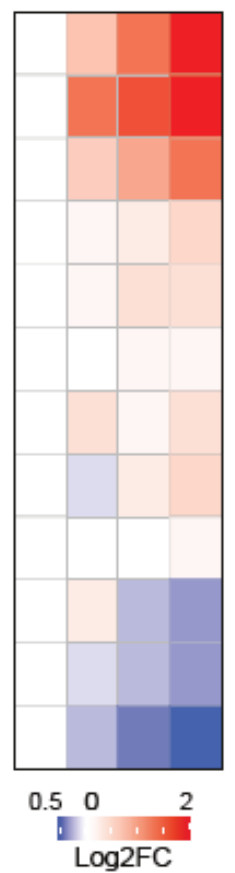

B

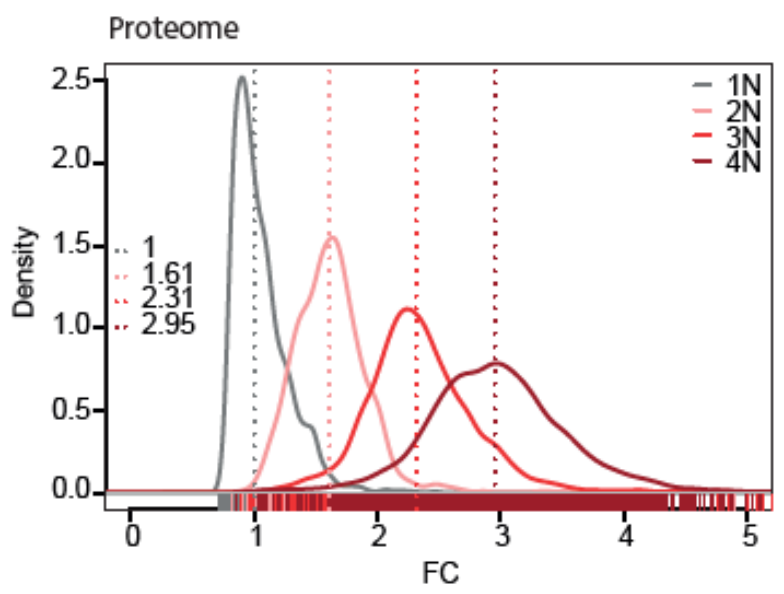

$\mathrm{D}$

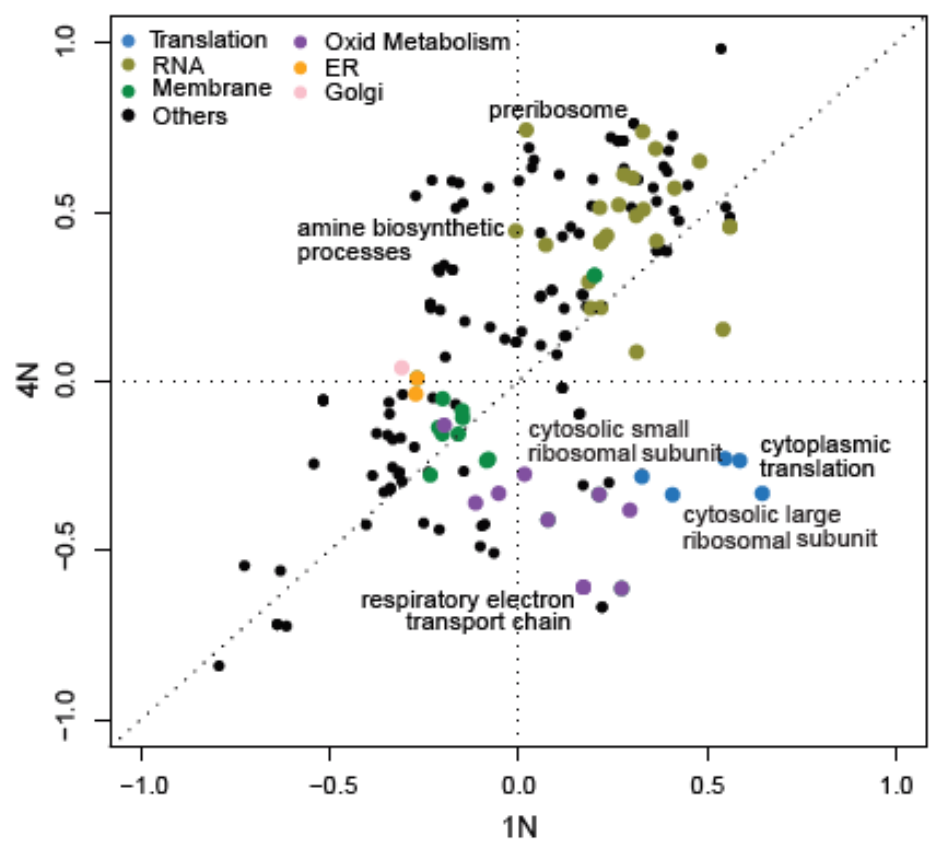

Figure 1. Proteome changes in response to increasing ploidy. (A) Schematic depiction of the strategy used for proteome analysis.(B) Proteome scaling with increasing ploidy. The values were normalized to the internal standard, with haploids set with a median at 1. 3109 were quantified in all ploidies. (C) Validation of the abundance changes of selected proteins. Left: relative proteome changes with ploidy normalized to $1 \mathrm{~N}$; middle: representative immunoblot of the selected candidates; right: Quantification of the protein abundance fold changes (FC) as determined by three biological replicates of immunoblotting, normalized to $1 \mathrm{~N}$. (D) Two-dimensional pathway enrichment analysis compares the proteome data of $1 \mathrm{~N}$ and $4 \mathrm{~N}$ cells. Each dot represents a pathway as determined by GO or KEGG databases. Only pathways significantly deregulated with respect to internal standard are depicted, Benjamini-Hochberg FDR $<0.02$. Related pathways are color-coded. 
Progression through the cell cycle was slightly reduced in tetraploids, as shown previously (7) (Extended Data 1E).

To assess the global proteome changes in strains of different ploidy, we used stable isotope labeling of amino acids in cell culture (SILAC) using an external spike-in standard consisting of a heavy lysine (Lys8)-labeled SILAC yeast standard, followed by liquid chromatography-tandem mass spectrometry (23). The heavy-labeled standard represented an equal protein mix from cells of different ploidy and was added to equal number of cells of different ploidy (Fig. 1A). The measurements provided quantitative information for $70 \%$ of all verified open reading frames in each strains with high correlation between independent experiments; this was also confirmed by principal component analysis (Extended Data 2A, B). 3109 protein groups quantified more than two times in three measurements were used to calculate the relative protein abundance in cells of each ploidy relative to the SILAC standard for normalization. This showed that the amount of proteins per cell increased with ploidy, but it did not scale linearly (Fig. 1B, $2 \mathrm{~N}: 1.61,3 \mathrm{~N}: 2.31,4 \mathrm{~N}: 2.95$ ). This "ploidy-specific protein scaling" (PSS) was validated by independent measurements of protein concentration and confirmed that the relative protein abundance per genome indeed decreases with increasing ploidy (Extended Data 2C, D).

While most proteins followed the PSS trend, we found that several proteins were differentially regulated by ploidy (ploidy-dependent regulation, PDR). We calculated the $\log 2$ fold change relative to the SILAC standard and normalized the data by shifting the median of individual ploidies to 0 (Supplementary Data 1, the data can be visualized via a web-based application PloiDEx). This is not an artifact of SILAC or the normalization, as immunoblotting of 12 selected proteins matched well with the proteome results (Fig. 1C). To identify the differentially regulated proteins, we took two approaches: (1) we calculated $4 \mathrm{~N} / 1 \mathrm{~N}$ ratio of protein abundance, and (2) classified proteins with a consistent abundance change across all ploidies (Extended Data 3A-D, for details see Methods). Among the proteins with $\pm 2 \mathrm{FC}$ across ploidies, we found several upregulated cell wall integrity proteins (e.g. Cbk1, Prt1, Emw1, Fks1), whereas multiple mitochondrial (Isu2, Tma17, Tim13) and ribosomal (Rpl13A, Rpl31B) proteins were downregulated. Similar changes in protein abundance were observed also when yeast strains of a different genetic background were used, or when cultured in different media (Extended Data 3E-H). Moreover, PDR was not due to an increased volume of polyploid cells, as shown by analysis of two haploid mutants with altered cell size: $\operatorname{cln} 3 \Delta$ that lacks a G1 cyclin, and a respiration deficient $r h o^{\circ}$ mutant (Extended Data 4A, B). While the volume of these haploid mutants was comparable to that of wild type diploids and triploids (Extended Data 4B, C), the protein abundance of selected candidates did not correspond with the changes observed in polyploids (Extended Data 4D). Thus, ploidy changes induce differential regulation of several specific proteins.

Previous analyses of mRNA expression showed only marginal changes that could not explain the proteome changes described above $(2,9,22)$. To confirm these findings in our strains, we used comparative differential transcription analysis (cDTA, (24)). mRNA was extracted from $S$. cerevisiae cells of different ploidy $(1 \mathrm{~N}, 2 \mathrm{~N}, 3 \mathrm{~N}$, $4 \mathrm{~N}$ ) labeled with 4-thiouracil (4tU) and mixed it with mRNA of the distantly related haploid fission yeast Schizosaccharomyces pombe labeled with 4sU (4-thiouridin) as an internal standard (Extended Data $5 \mathrm{~A},(24))$. This allowed us to obtain data on abundance changes for 5656 mRNAs. Comparison of mRNA abundance in cells of different ploidy showed similar ploidy-specific scaling as observed for protein abundance (Extended Data 5B). We then transformed the values to $\log 2 \mathrm{FC}$ and normalized them by shifting the median of the distribution to 0 . In agreement with previously published results $(2,9,22)$, the expression of vast majority of mRNAs was not differentially expressed in polyploid cells (Data File 2, PloiDEx). Only 13 mRNAs change significantly with ploidy ( \pm 2 FC), including factors involved in plasma membrane and cell wall synthesis (Extended Data 5C,D), in agreement with previous findings $(9,22)$. Remarkably, qPCR analysis of selected factors whose protein levels were differentially regulated by ploidy confirmed that the changes were not present in the transcriptome (Extended Data 5E,F). Thus, the ploidy-dependent regulation of protein abundance occurs posttranscriptionally.

We next asked which pathways are differentially regulated in response to ploidy changes. Two-dimensional pathway enrichment comparison revealed striking ploidy-dependent down-regulation of pathways related to cytoplasmic ribosomes, translation, and mitochondrial respiration (Fig. 1D). Gene set enrichment analysis (GSEA) of the significantly deregulated proteins also confirmed that "ribosome biogenesis" and "cytoplasmic translation" were repressed with increasing ploidy, whereas vesicle trafficking and intracellular transport were activated (Extended Data 6A, B, Supplementary Data 3). Few pathways were differentially regulated at the mRNA level and there was no overlap with the proteome (Extended Data 6C, D), further confirming the low correlation between ploidy-dependent transcriptome and proteome changes. Proteome analysis showed that the respiratory electron transport chain was reduced. Consistently, the abundance of Rtg2, which plays a central role in retrograde signaling of mitochondrial dysfunction to the nucleus, was increased in cells of higher ploidy because of the stabilization of this protein (Fig. 1C, Extended Data 7A, B). Polyploid cells also proliferated poorly on media with non-fermentable carbon source or in the presence of the oxidant diamide (Extended Data 7C). This suggests ploidy-specific deregulation of mitochondrial function, in line with recent findings in pathogenic yeast Candida albicans (25).

Strikingly, "cytoplasmic translation" and "ribosome biogenesis" were strongly reduced with increasing ploidy (Fig. 1D, Extended Data 8A). We focused on this aspect, because it could explain the observed allometric PSS of protein content (Fig. 1B, Extended Data 2C, D). No mRNA changes could explain the altered abundance of ribosomal proteins, thus, we hypothesized that production of rRNA is reduced with increasing ploidy. Indeed, qRT-PCR revealed reduced abundance of $25 \mathrm{~S}$ and $5.8 \mathrm{~S}$ rRNA in polyploid cells (Fig. 2A). We used pulse-labeling with puromycin, an amino acid analog that is incorporated into the nascent polypeptide chain and serves as a proxy for translational efficiency. This showed that the relative translation rate increased with ploidy when equal number of cells was loaded, but the increase was not linear, in agreement with proteome quantification; the puromycin incorporation appeared to be constant when equal amount of protein was loaded (Fig. 2B-D, Extended Data 8B). 
A
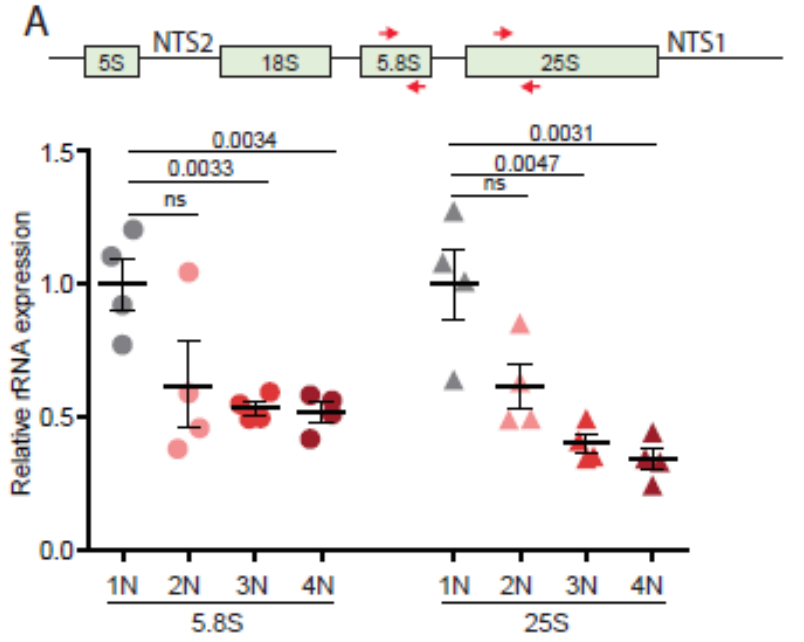

E

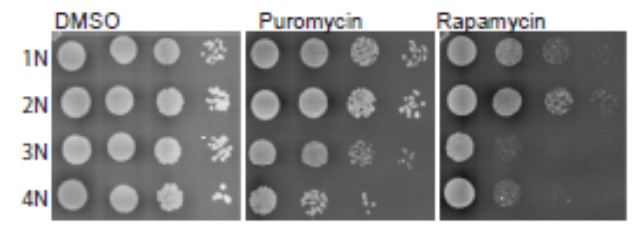

$\mathrm{F}$

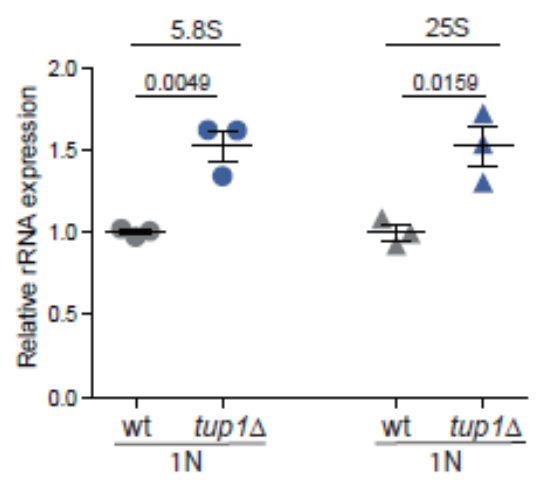

B

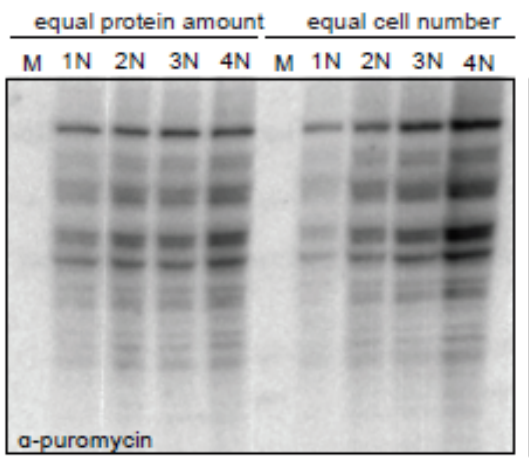

C

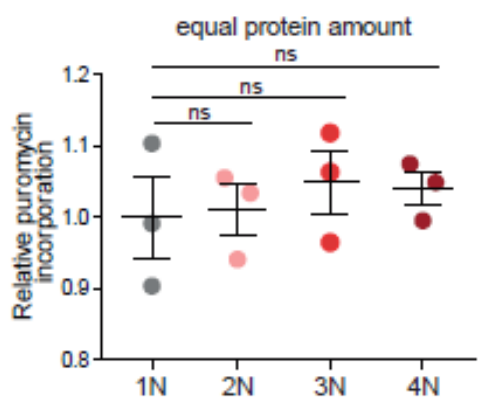

D $\frac{\mathrm{M} \quad 1 \mathrm{~N} 2 \mathrm{~N} \quad 3 \mathrm{~N} 4 \mathrm{~N}}{\mathrm{M} 1 \mathrm{~N} 2 \mathrm{~N} 3 \mathrm{~N} \quad 4 \mathrm{~N}}$
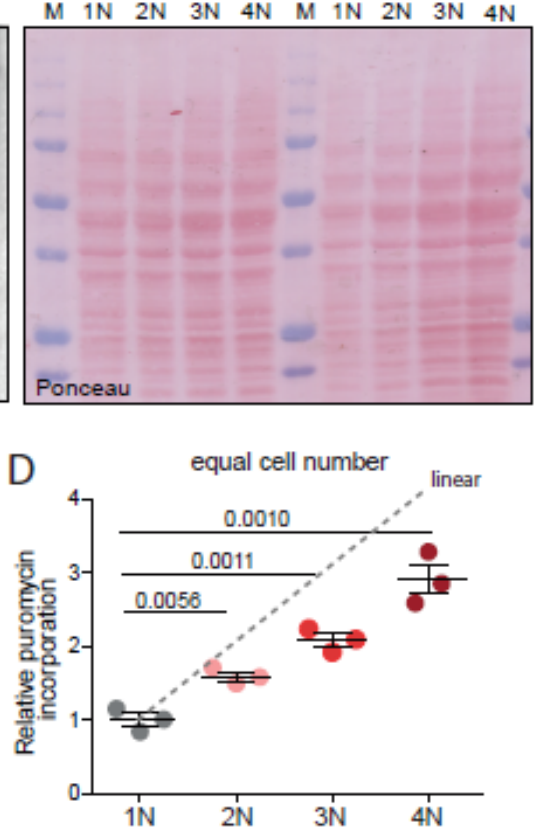

G

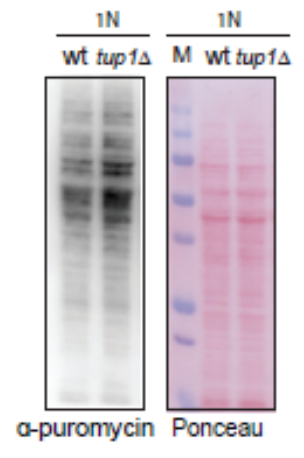

$\mathrm{H}$

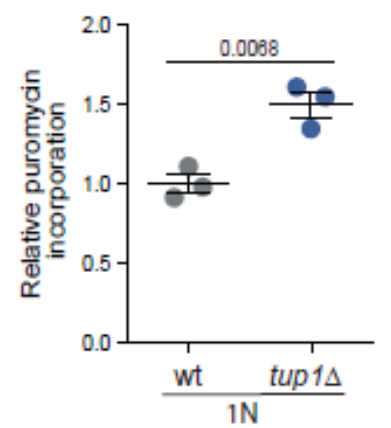

Figure 2. Translation and ribosome biogenesis are downregulated in cells with increased ploidy. (A) Quantification of rRNA abundance in cells of different ploidy. qRT-PCR was used in four independent experiments, the values were normalized to the expression of the housekeeping gene ACT1. Mean with SEM is shown. Top: schematics of the primers' localization. (B) Puromycin incorporation in cells of different ploidy. Two types of loading were applied: left: equal amount of protein lysates were loaded, right: lysates from equal cell number was loaded. Ponceau staining was used as a loading control. (C) (D) Quantification of the relative puromycin incorporation calculated from three independent experiments; means with SEM are shown. (E) Sensitivity of cells of different ploidy to puromycin and rapamycin treatment. (F) Quantification of rRNA in haploid wt cells and in mutants lacking TUP1. The experiment was performed as in (A). (G) Representative immunoblotting of puromycin incorporation in haploid wt and tupl $\Delta$ cells. (H) Quantification of puromycin incorporation from three independent biological replicates. Equal amount of protein lysate was loaded, Ponceau staining was used for the loading control. Means with SEM are shown. 
The cells of higher ploidy were more sensitive to puromycin, an inhibitor of translation, as well as to rapamycin, an inhibitor of the master regulator of cellular metabolism mTOR, supporting the notion that the translational regulation is compromised via altered mTOR signaling (Fig. 2E).

What mechanism controls the reduced ribogenesis and translation in cells with higher ploidy? Because rRNA levels were reduced, we hypothesized that rDNA transcription decreases with increasing ploidy. We therefore examined all differentially regulated proteins for motives commonly found in repressors or for annotation indicating involvement in rRNA transcriptional repression. We identified Tup1, a protein whose human homolog Tle1 was previously shown to mediate rRNA repression (26). Accordingly, deletion of TUPl in $1 \mathrm{~N}$ strains caused no changes in the abundance of several candidate mRNAs (Extended Data 8C), but lead to a significant increase in rRNA abundance and an increase in puromycin incorporation rates (Fig. 2F-H).

Ribosome biogenesis in eukaryotes is regulated via the mTOR pathway. Strikingly, treatment of haploid cells with rapamycin for $1 \mathrm{~h}$ increased Tup1 abundance to levels observed in tetraploids (Fig. 3A, B), clearly linking the regulation of Tup1 and rRNA gene expression to the mTOR pathway. In budding yeast, Tor1 phosphorylates the kinase Sch9, a yeast homolog of human P70-S6K, to promote ribosome biogenesis and protein synthesis. We asked whether Tor1-Sch9 regulates rDNA expression via Tup1. Haploid cells lacking Sch9 accumulated Tup1 independently of rapamycin treatment, suggesting that Tor1 negatively regulates Tup1 via Sch9 kinase (Fig. 3C). To test whether Tup1 is a downstream target of Sch9, we constructed a haploid strain with the analog-sensitive allele of Sch9 (Sch9-as, (27)). Inhibition with the ATP analog 1NM-PP1 increased the abundance of Tup1 and reduced its phosphorylation, as documented by altered migration in the Phos-TAG gel (Fig. 3D, Extended Data 8D). Finally, in haploid cells, loss of TUP1 lead to constitutively high rRNA levels, while loss of $\mathrm{SCH} 9$ reduced them (Fig. 3E). We asked whether the Tor1-Sch9 activity declines with increasing ploidy. Indeed, we observed decreased phosphorylation of Sch9 and Tup1, as judged by a reduced shift in migration on Phos-TAG gel, similar to mTOR inhibition with rapamycin (Fig. 3F, G, Extended Data 8E).Cyclohexamide shut off revealed that the stability of Tup1 increased in tetraploid cells, as well as in haploid cells lacking $S C H 9$ (Fig. 3H, I). We conclude that Tor1- Sch9 activity is reduced with increasing ploidy, which leads to accumulation of the negative regulator of rDNA expression Tup1. We propose that this novel signaling pathway is responsible for the ploidy-specific proteome scaling.

We asked whether downregulation of translation and ribosome biogenesis occurs also in human tetraploid cells. To this end, we induced cytokinesis failure by treatment with the actin inhibitor dihydrocytochalasin D (DCD) in human diploid colorectal cancer cell line HCT116. This treatment induced formation of binucleated tetraploid cells (28). We also isolated a near-tetraploid cell line HPT2 (HCT116 Post Tetraploid) derived from a single HCT116 cell after WGD (Extended Data 9A)(17). Chromosome numbers and cell size increased with WGD as expected, as did the population heterogeneity (Fig. 4A, Extended Data 9B, C). While ribosomal protein expression was doubled in freshly formed binucleated tetraploids, four of six ribosomal proteins tested were reduced in HPT2 (Fig. 4B). Analysis of the previously obtained transcriptome of HPT2 confirmed that these changes were due to posttranscriptional regulation (17). Puromycin incorporation during translation was increased in binucleated tetraploids immediately after WGD, but was reduced in HPT2 below the diploid levels (Fig. 4C). This reduction was likely mediated via the mTORC1-S6K(Sch9)-Tle1(Tup1) pathway, as Tle1 abundance was elevated and mTORC1 activity, estimated by phosphorylation of S6K, was reduced in HPT2 but not in binucleated tetraploids (Fig. 4D-F, Extended Data 9D). Phosphorylation of the translation initiation factor $2, \alpha$ subunit (eIF2 $\alpha$ ) was not changed, suggesting that translational initiation was not affected by ploidy (Extended Data S9E). Finally, the expression of rRNA was reduced in the posttetraploid cells compared with the parental control (Fig. 4G). Thus, reduced mTORC1 activity and, in turn, reduced translation represents a conserved cellular response to increased ploidy and may be one of the major factors for the observed allometric scaling of protein levels with increased ploidy.

This first proteome analysis in cells of different ploidy reveals striking ploidy-dependent proteome changes. First, we showed that protein abundance does not increase linearly with ploidy. Rather, it increases in accordance with allometric scaling theory where metabolic rate is predicted to scale with body size with an exponent close to $3 / 4$ $(29,30)$, or, alternatively, with surface area with a scaling exponent of $2 / 3$ (31). The observed PDS may have critical implication for cell physiology. Because the genome content and cellular volume increases linearly $(2,9)$, allometric proteome scaling leads to cell dilution and a reduced ratio of DNA-binding proteins to DNA. Second, we show that many individual proteins are differentially regulated in response to ploidy. We call this phenomenon ploidy-dependent regulation - PDR. Our findings may help explain phenotypes of tetraploid cells that were difficult to reconcile with the minor changes observed in transcriptome and thus provide new hypotheses for mechanisms underlying ploidy-specific effects.

Strikingly, proteins related to ribosomes and translation were downregulated with increasing ploidy. The observed PDR of ribosome biosynthesis and translation also provided an explanation for the allometric proteome scaling, which we propose is regulated by the mTORC1-Sch9-Tup1 axes (Fig. 4H). Tup1 is a general transcription repressor that affects promoter accessibility (32). We found that Tup1 becomes stabilized by reduced Tor1-Sch9 activity in polyploids, resulting in reduced rRNA synthesis. The abundance of rRNA is limiting for ribosome biogenesis and thus the regulation of rRNA synthesis is central to overall ribosome synthesis. In agreement, the Tup1 homolog in humans, TLE1, previously shown to regulate rRNA expression (26), is also stabilized in tetraploid human cells.

What triggers reduced rDNA expression in polyploids? Ribosome biosynthesis is tightly regulated in response to environmental stress, and indeed stressors such as heat shock, osmotic shock, and nutrients deprivation reduce the expression of ribosomal proteins (33) and rRNAs (34). The reduced translation and rDNA expression could be a direct fitness cost of increased cell volume. The decreased surface-to-volume ratio may impair nutrient uptake, which in turn reduces mTOR activity and forces downregulation of protein synthesis. 
bioRxiv preprint doi: https://doi.org/10.1101/2021.05.06.442919; this version posted May 21, 2021. The copyright holder for this preprint (which was not certified by peer review) is the author/funder. All rights reserved. No reuse allowed without permission.

A

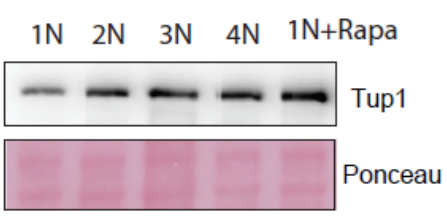

B

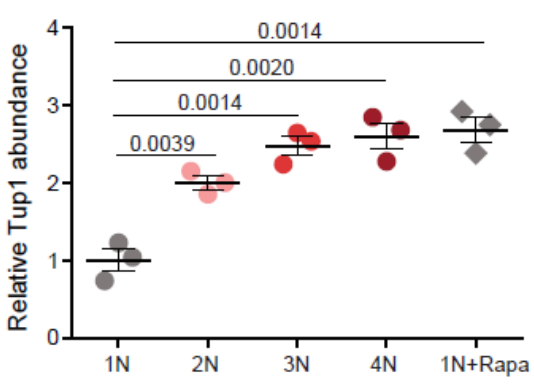

C

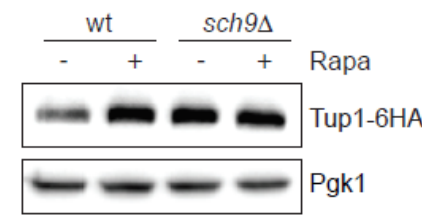

D

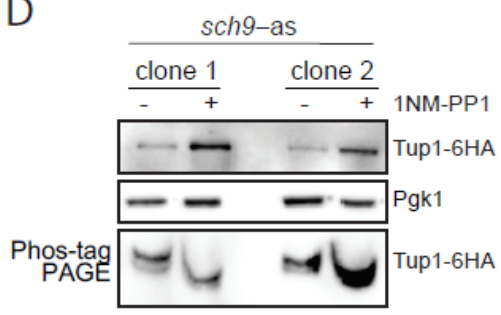

$\mathrm{E}$

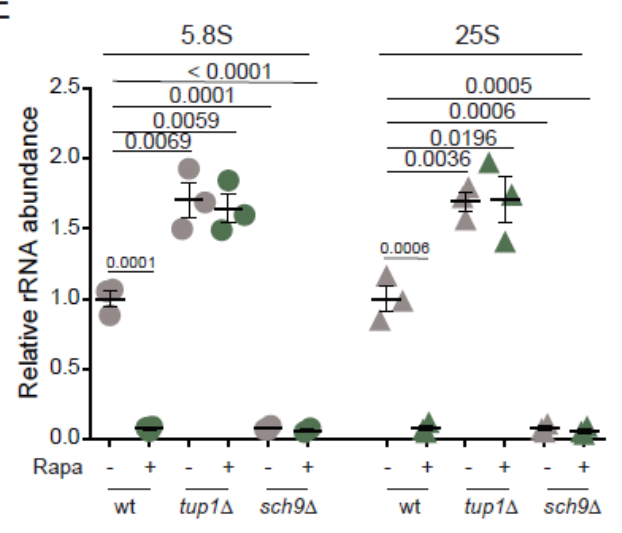

$\mathrm{F}$

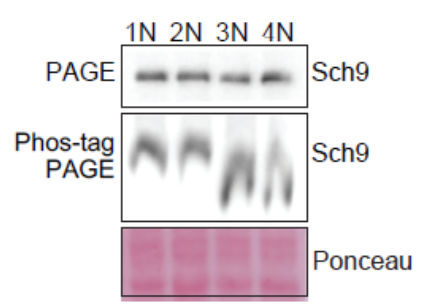

G

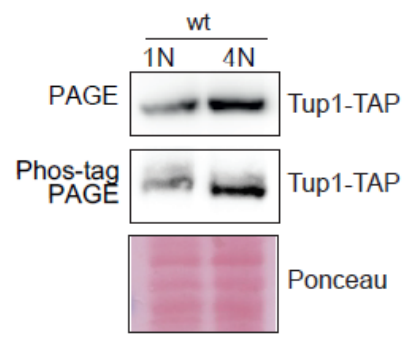

$\mathrm{H}$
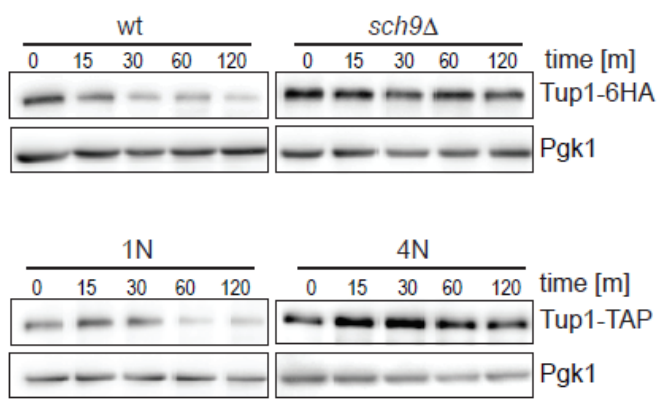

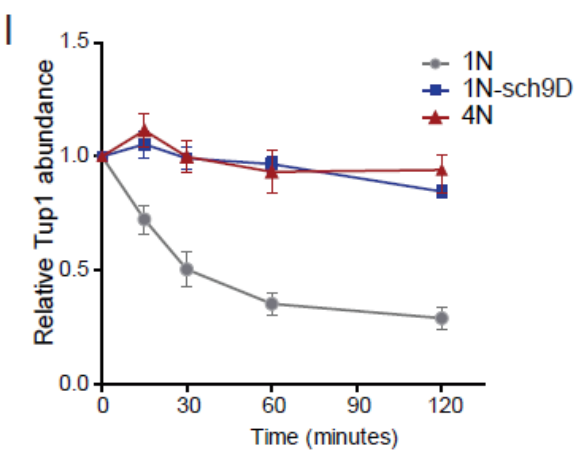

Figure 3. Regulation of rRNA expression in polyploid cells via Tor1-Sch9-Tup1 signaling pathway. (A) Relative abundance of Tup1 in cells of different ploidy compared to haploid cells treated with rapamycin. (B) Quantification of three independent experiments as in (A). Mean with SEM is shown. (C) Representative immunoblot showing the abundance of Tup1 in wt and sch9s haploid cells treated with rapamycin. (D) Representative immunoblot showing the abundance of Tup1 in sch9-as haploid cells with and without the ATP analogue 1NM-PP1. Two different clones were tested. (E) qRT-PCR quantification of rRNA abundance in cells lacking Sch9 and Tup1, with and without rapamycin treatment (Rapa). Means and SEM of three independent experiments are shown. (F) Representative immunoblotting of Sch9 on PAGE and Phos-TAG gel in cells of different ploidy. (G) Representative immunoblotting of Tup1 on PAGE and Phos-TAG gel in cells of different ploidy. (H) Representative immunoblotting of Tup1 after cyclohexamide shut off. Different time points were collected and equal amount of lysate was loaded. (I) Quantification of three independent experiments from (H). Means and SEM are shown. 
Tetraploid yeast cells are sensitive to starvation (21) and often undergo ploidy reduction $(3,8,19)$. Finally, stable tetraploid cells evolved for 1000 generations were smaller than the original cells, although they retained the tetraploid karyotype (35). These findings suggest that cell size might be a limiting factor causing strong selection to reduce ploidy or cell size, or both. Additionally, membrane stress due to abnormally large volume, and mitochondrial defect are known to alter mTOR activity and therefore may contribute to the observed PDR and PSS (36).

Another possibility is that downregulation of rDNA transcription could enhance adaptation to increased ploidy to prevent aberrant homologous recombination within the transcriptionally active rDNA repeats and R-loop formation. This hypothesis is alos supported by previous finding that the kinase Sch9 is required for the evolution of genomically stable yeast tetraploids (35). Genomic stability is impaired in cells with higher ploidy, and recombination rates increase significantly in budding yeast tetraploids (9). Because formation of R-loops threatens genome stability and increases recombination, frequent transcription of rDNA could be fatal to already compromised genomic stability in tetraploid cells.

Finally, an important observation is that PDR occurs largely on proteome level, via changes in protein stability. Proteome changes can occur rapidly and likely respond to more subtle environmental changes. Perhaps increased stress or prolonged in vitro evolution would be required to rewire the transcriptional patterns. Interestingly, many of the same genes and pathways identified here are altered at the transcriptional level after autopolyploidization in plants (37). Ribosomal and mitochondrial genes are also preferentially lost during evolution after whole genome duplication in Salmonidae (38). Together, these findings suggest conserved cellular changes triggered by increased ploidy that enable global metabolic adjustments to ensure survival of cells after whole genome doubling.

\section{References}

1. Storchova, Z. Pellman, D. From polyploidy to aneuploidy, genome instability and cancer. Nat Rev Mol Cell Biol 5, 45-54, doi:10.1038/ nrm1276 (2004).

2. Galitski, T., Saldanha, A. J., Styles, C. A., Lander, E. S. Fink, G. R. Ploidy regulation of gene expression. Science 285, 251-254, doi:10.1126/science.285.5425.251 (1999).

3. Gerstein, A. C., Chun, H. J., Grant, A. Otto, S. P. Genomic convergence toward diploidy in Saccharomyces cerevisiae. PLoS Genet 2 , e145, doi:10.1371/journal.pgen.0020145 (2006).

4. Di Talia, S., Skotheim, J. M., Bean, J. M., Siggia, E. D. Cross, F. R. The effects of molecular noise and size control on variability in the budding yeast cell cycle. Nature 448, 947-951, doi:10.1038/ nature06072 (2007).

5. Schoenfelder, K. P. Fox, D. T. The expanding implications of polyploidy. J Cell Biol 209, 485-491, doi:10.1083/jcb.201502016 (2015).

6. Weiss, R. L., Kukora, J. R. Adams, J. The relationship between enzyme activity, cell geometry, and fitness in Saccharomyces cerevisiae. Proc Natl Acad Sci U S A 72, 794-798, doi:10.1073/pnas.72.3.794 (1975).

7. Mable, B. K. Ploidy evolution in the yeast Saccharomyces cerevisiae: a test of the nutrient limitation hypothesis. J Evol Biol 14, 157-170, doi:10.1046/ j.1420-9101.2001.00245.x (2001).
8. Mayer, V. W. Aguilera, A. High levels of chromosome instability in polyploids of Saccharomyces cerevisiae. Mutat Res 231, 177-186, doi:10.1016/ 0027-5107(90)90024-x (1990).

9. Storchova, Z. et al. Genome-wide genetic analysis of polyploidy in yeast. Nature 443, 541-547, doi:10.1038/nature05178 (2006).

10. Comai, L. The advantages and disadvantages of being polyploid. Nat Rev Genet 6, 836-846, doi:10.1038/nrg1711 (2005).

11. Van de Peer, Y., Mizrachi, E. Marchal, K. The evolutionary significance of polyploidy. Nat Rev Genet 18, 411-424, doi:10.1038/ nrg.2017.26 (2017).

12. Zack, T. I. et al. Pan-cancer patterns of somatic copy number alteration. Nat Genet 45, 1134-1140, doi:10.1038/ng.2760 (2013).

13. Bielski, C. M. et al. Genome doubling shapes the evolution and prognosis of advanced cancers. Nat Genet 50, 1189-1195, doi:10.1038/ s41588-018-0165-1 (2018).

14. Fujiwara, T. et al. Cytokinesis failure generating tetraploids promotes tumorigenesis in p53-null cells. Nature 437, 1043-1047, doi:10.1038/ nature04217 (2005).

15. Storchova, Z. Ploidy changes and genome stability in yeast. Yeast 31, 421-430, doi:10.1002/yea.3037 (2014).

16. Dewhurst, S. M. et al. Tolerance of whole-genome doubling propagates chromosomal instability and accelerates cancer genome evolution. Cancer Discov 4, 175-185, doi:10.1158/2159-8290.CD-13-0285 (2014).

17. Kuznetsova, A. Y. et al. Chromosomal instability, tolerance of mitotic errors and multidrug resistance are promoted by tetraploidization in human cells. Cell Cycle 14, 2810-2820, doi:10.1080/ 15384101.2015. 1068482 (2015).

18. Nano, M. et al. Cell-Cycle Asynchrony Generates DNA Damage at Mitotic Entry in Polyploid Cells. Curr Biol 29, 3937-3945 e3937, doi:10.1016/j.cub.2019.09.041 (2019).

19. Selmecki, A. M. et al. Polyploidy can drive rapid adaptation in yeast. Nature 519, 349-352, doi:10.1038/nature14187 (2015).

20. Otto, S. P. The evolutionary consequences of polyploidy. Cell 131, 452-462, doi:10.1016/j.cell.2007.10.022 (2007).

21. Andalis, A. A. et al. Defects arising from whole-genome duplications in Saccharomyces cerevisiae. Genetics 167, 1109-1121, doi:10.1534/genetics.104.029256 (2004).

22. Wu, C. Y., Rolfe, P. A., Gifford, D. K. Fink, G. R. Control of transcription by cell size. PLoS Biol 8, e1000523, doi:10.1371/ journal.pbio. 1000523 (2010).

23. Nagaraj, N. et al. System-wide perturbation analysis with nearly complete coverage of the yeast proteome by single-shot ultra HPLC runs on a bench top Orbitrap. Mol Cell Proteomics 11, M111 013722 , doi:10.1074/mcp.M111.013722 (2012).

24. Miller, C. et al. Dynamic transcriptome analysis measures rates of mRNA synthesis and decay in yeast. Mol Syst Biol 7, 458, doi:10.1038/ msb.2010.112 (2011).

25. Thomson, G. J. et al. Metabolism-induced oxidative stress and DNA damage selectively trigger genome instability in polyploid fungal cells. EMBO J 38, e101597, doi:10.15252/embj.2019101597 (2019).

26. Ali, S. A. et al. Transcriptional corepressor TLE1 functions with Runx2 in epigenetic repression of ribosomal RNA genes. Proc Natl Acad Sci U S A 107, 4165-4169, doi:10.1073/pnas.1000620107 (2010).

27. Huber, A. et al. Sch9 regulates ribosome biogenesis via Stb3, Dot6 and Tod6 and the histone deacetylase complex RPD3L. EMBO J 30, 3052-3064, doi:10.1038/emboj.2011.221 (2011).

28. Kuffer, C., Kuznetsova, A. Y. Storchova, Z. Abnormal mitosis triggers p53-dependent cell cycle arrest in human tetraploid cells. Chromo- 

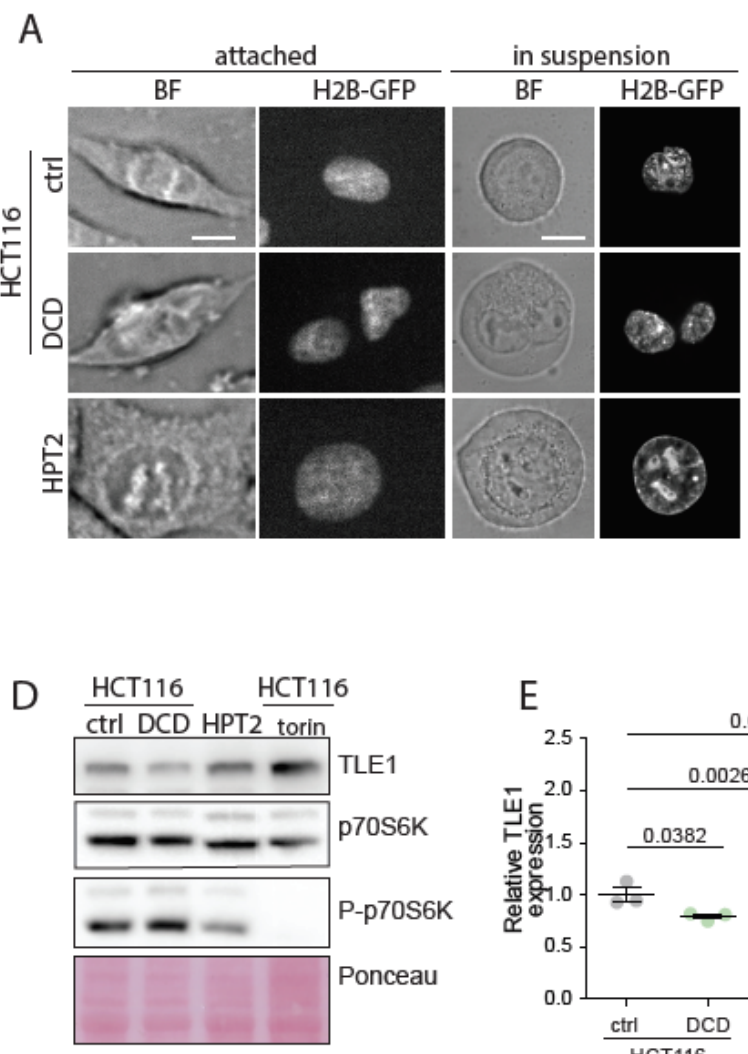

$\mathrm{F}$

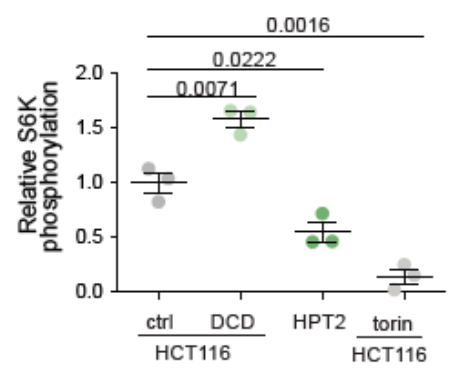

G
B
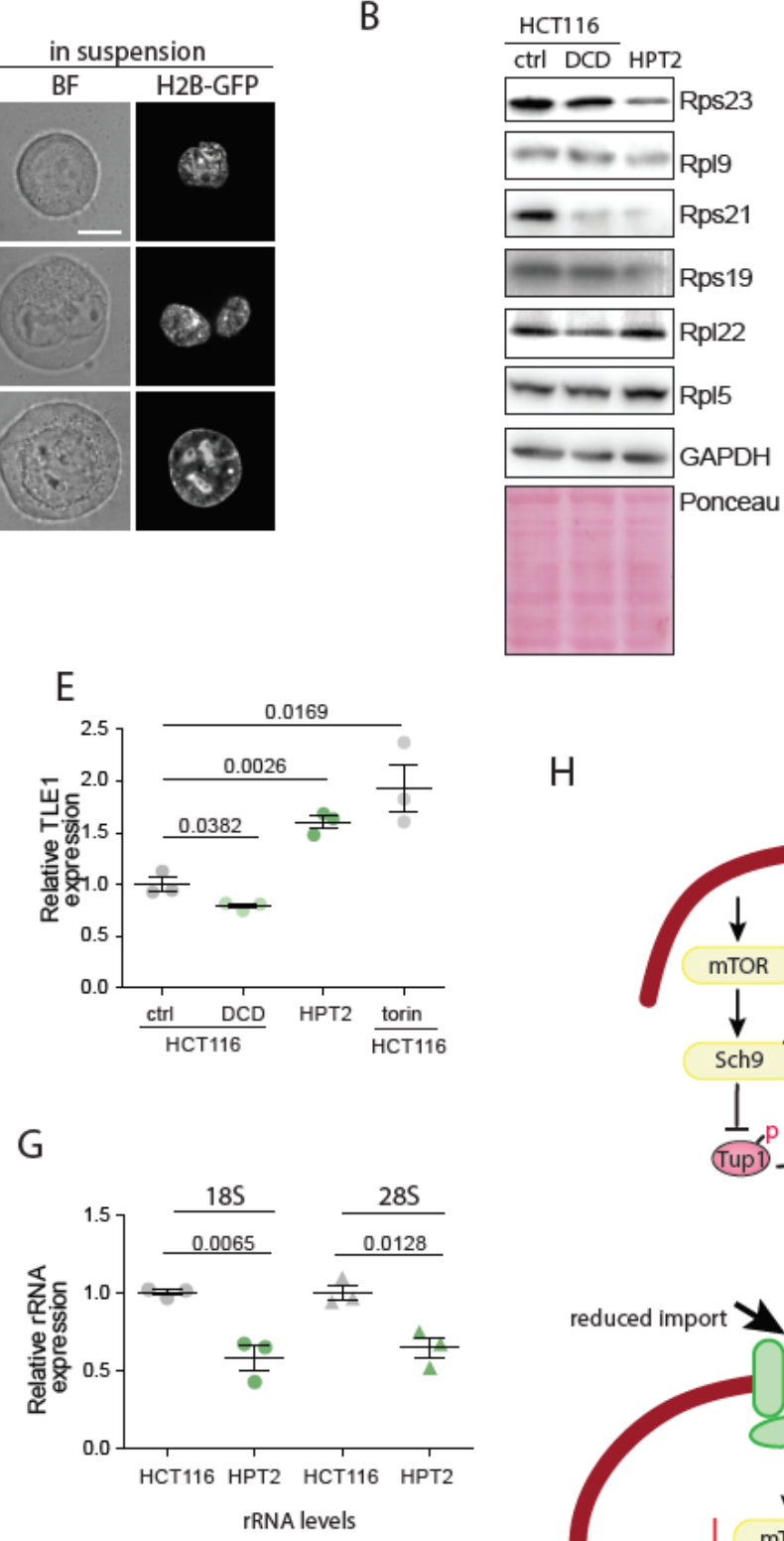

$\mathrm{H}$
C
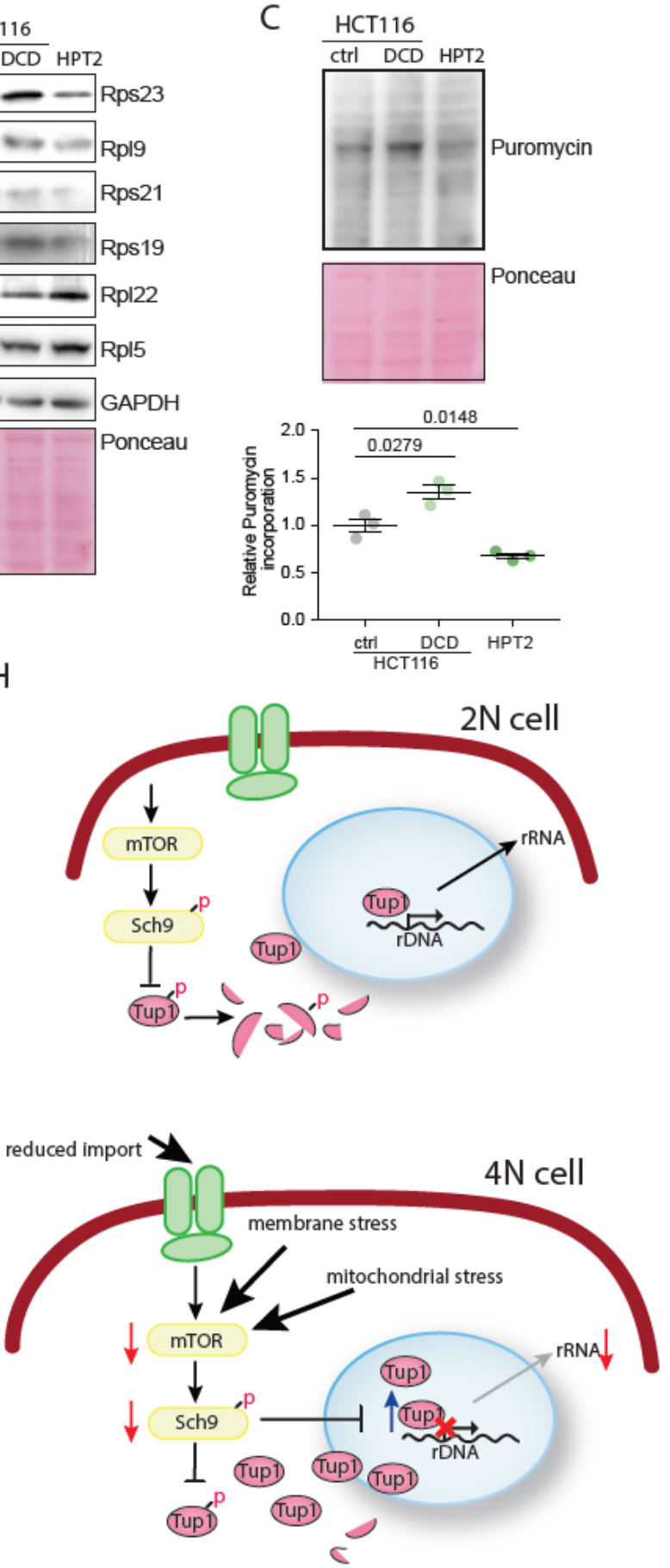

Figure 4. Downregulation of ribosomal proteins and translation in human near-tetraploid cells. (A) Cell size changes in response to altered ploidy. ctrl - untreated HCT116, DCD - HCT116 treated with dyhydrocytochalasin D to induce cytokinesis failure and subsequent tetraploidy; HPT2 - HCT116derived near-tetraploid cell line. (B) Abundance of ribosomal proteins in response to altered ploidy. ctrl - untreated HCT116, DCD - HCT116 treated with dyhydrocytochalasin D to induce cytokinesis failure and subsequent tetraploidy; HPT2 - HCT116- derived near-tetraploid cell line. (C) Puromycin incorporation in diploid, newly made tetraploid HCT116 compared to near-tetraploid HPT2. Bottom: quantification of the relative puromycin incorporation. (D) Representative immunoblotting of Tle1 and S6K in human cells of different ploidy. (E) Quantification of Tle1, three independent experiments were analyzed. (F) Quantification of S6K from three independent experiments. (G) rRNA abundance in cells of different ploidy. Mean and SEM of three independent experiments is shown. (H) Schematic model of the translation regulation in polyploid cells. 
soma 122, 305-318, doi:10.1007/s00412-013-0414-0 (2013).

29. West, G. B., Woodruff, W. H. Brown, J. H. Allometric scaling of metabolic rate from molecules and mitochondria to cells and mammals. Proc Natl Acad Sci U S A 99 Suppl 1, 2473-2478, doi:10.1073/ pnas.012579799 (2002).

30. Savage, V. M., Deeds, E. J. Fontana, W. Sizing up allometric scaling theory. PLoS Comput Biol 4, e1000171, doi:10.1371/ journal.pcbi. 1000171 (2008).

31. Hou, C. et al. Energy uptake and allocation during ontogeny. Science 322, 736-739, doi:10.1126/science.1162302 (2008).

32. Tam, J. van Werven, F. J. Regulated repression governs the cell fate promoter controlling yeast meiosis. Nat Commun 11, 2271, doi:10.1038/s41467-020-16107-w (2020).

33. Gasch, A. P. et al. Genomic expression programs in the response of yeast cells to environmental changes. Mol Biol Cell 11, 4241-4257, doi:10.1091/mbc.11.12.4241 (2000).

34. Laferte, A. et al. The transcriptional activity of RNA polymerase I is a key determinant for the level of all ribosome components. Genes Dev 20, 2030-2040, doi:10.1101/gad.386106 (2006).

35. Lu, Y. J., Swamy, K. B. Leu, J. Y. Experimental Evolution Reveals Interplay between Sch9 and Polyploid Stability in Yeast. PLoS Genet 12, e1006409, doi:10.1371/journal.pgen.1006409 (2016).

36. Kawai, S. et al. Mitochondrial genomic dysfunction causes dephosphorylation of Sch9 in the yeast Saccharomyces cerevisiae. Eukaryot Cell 10, 1367-1369, doi:10.1128/EC.05157-11 (2011).

37. Song, Q., Ando, A., Jiang, N., Ikeda, Y. Chen, Z. J. Single-cell RNAseq analysis reveals ploidy-dependent and cell-specific transcriptome changes in Arabidopsis female gametophytes. Genome Biol 21, 178, doi:10.1186/s13059-020-02094-0 (2020).

38. Gillard, G. B. et al. Comparative regulomics reveals pervasive selection on gene dosage following whole genome duplication. bioRxiv, 2020.2007.2020.212316, doi:10.1101/2020.07.20.212316 (2020).

39. Cox, J. Mann, M. MaxQuant enables high peptide identification rates, individualized p.p.b.-range mass accuracies and proteome-wide protein quantification. Nat Biotechnol 26, 1367-1372, doi:10.1038/ nbt.1511 (2008).

40. Sun, M. et al. Comparative dynamic transcriptome analysis (cDTA) reveals mutual feedback between mRNA synthesis and degradation. Genome Res 22, 1350-1359, doi:10.1101/gr.130161.111 (2012).

\section{Acknowledgments}

We thank the members of Storchova lab, as well as Simen Rød Sandve and Johannes M. Herrmann for discussions and support. We thank Sara Bernhard for help with human tetraploid cells and Robbie Loewith for providing the Sch9-as coding plasmid. Funding: GY is a visiting scientist funded by Alexander von Humboldt Foundation (Georg Foster Stipend).

\section{Author Contributions}

GY was involved in conceptualization, investigation, validation and funding acquisition, PM and MR were involved in investigation, formal analysis and data curation, AW contributed resources and investigation, DS and NK were involved in investigation, formal analysis and data curation, MM and PC were involved in conceptualization, supervision and funding acquisition, $\mathrm{ZS}$ was involved in conceptualization, writing, supervision and funding acquisition.

\section{Declaration of Interests}

The authors declare no competing interests.

\section{Data and materials availability}

The proteome and transcriptome data sets are available from public repositories, see details in Supplementary Materials.

\section{Methods}

Yeast media and culture. YP medium containing $1 \%$ yeast extract, $2 \%$ bacto-peptone, $2 \%$ dextrose, galactose or glycerol, respectively, were used. The synthetic drop-out (SC) medium consists of $5 \mathrm{~g} / \mathrm{L}$ (NH4)2SO4, $2 \mathrm{~g} / \mathrm{L}$ KH2PO4, $0.5 \mathrm{~g} / \mathrm{L} \mathrm{MgSO}$ •7H2O, $0.1 \mathrm{~g} / \mathrm{L}$ $\mathrm{CaCl} 2 \cdot 2 \mathrm{H} 2 \mathrm{O}, 0.02 \mathrm{~g} / \mathrm{L}$ FeSO $4 \bullet 7 \mathrm{H} 2 \mathrm{O}, 0.01 \mathrm{~g} / \mathrm{L} \mathrm{ZnSO} 4 \bullet 7 \mathrm{H} 2 \mathrm{O}, 0.005$ $\mathrm{g} / \mathrm{L} \mathrm{CuSO} 4 \cdot 5 \mathrm{H} 2 \mathrm{O}, 0.001 \mathrm{~g} / \mathrm{L} \mathrm{MnCl} 2 \bullet 4 \mathrm{H} 2 \mathrm{O}, 1 \mathrm{~g} / \mathrm{L}$ yeast extract, 10 $\mathrm{g} / \mathrm{L}$ glucose, $0.5 \mathrm{~mL} / \mathrm{L} 70 \% \mathrm{H} 2 \mathrm{SO} 4200 \mu \mathrm{g} / \mathrm{ml}$. G418 or $100 \mu \mathrm{g} / \mathrm{ml}$ ClonNat or $300 \mu \mathrm{g} / \mathrm{ml}$ Hygromycin were supplemented in YPD for drug resistance selection. For drop dilution, YPD pates were supplemented with $10 \mu \mathrm{g} / \mathrm{ml}$ Benomyl, $10 \mathrm{ng} / \mathrm{ml}$ Rapamycin, $500 \mathrm{ng} / \mathrm{ml}$ Tunicamycin $25 \mu \mathrm{M}$ Puromycin, or $0.25 \mu \mathrm{M}$ Diamide. For measurements of the protein half-life, the indicated strains were grown exponentially in YPD, then Cycloheximide (CHX) was added to a final concentration of $50 \mu \mathrm{g} / \mathrm{mL}$ to inhibit protein synthesis, the point of which was defined as time zero. Cell aliquots were collected at the indicated time points for protein extraction and western blotting. For experiments employing the sch9-as allele, $10 \mathrm{nM}$ of 1NM-PP1 was used to inhibit the kinase activity of sch9-as.

Yeast strain construction. To obtain yeast isogenic strain of different ploidies suitable for analysis, we used the strains of S288C genetic background BY4741 and BY4742. LYS2 gene was deleted using xyz cassette to enable labeling with heavy lysine for SILAC experiments. BY4741 was transformed with a plasmid carrying the $\mathrm{HO}$ gene under the control of galactose-inducible promoter. Diploid strain was created by mating Mata and MAT $\alpha$ haploid strains. Upon induction of HO expression, single diploid colonies that become matingcompetent were selected and used for further mating to create triploid and tetraploid strain (Extended Data 1A). All strains were switch by $\mathrm{HO}$ expression to MATa mating type and the HO-expressing vector was removed by treatment with 5-FOA. Gene knockout strains were either retrieved from the available libraries deposit in public repository Euroscarf, or generated by homologous recombination using PCR products containing a drug cassette (kanMX6, Clonnat, hygMX) and $40 \mathrm{bp}$ sequences flanking the target gene. Taggedprotein strains were either retrieved from the publicly available library (Euroscarf) or generated by integrating a cassette containing a protein tag and a drug resistance cassette at the C-terminus. PCR products were transformed into a haploid strain, or into diploid strains and the heterozygous diploids were sporulated and dissected to select for haploids with drug resistance. Subsequently, the procedure of polyploid construction described above was performed. For construction of strains with analog-sensitive sch9 allele, pRS414::sch9as (T492G) was used (a gift from Robbie Loewith, University of Geneva). The 
list of used strains is in Supplementary Table 1.

Human cell line culture. HCT116 and the postetraploid derivative HPT2 were cultured in DMEM (Life Technologies) with 10\% fetal bovine serum (Sigma-Aldrich) and 1\% penicillin-streptomycinglutamine (Life Technologies). Cells were incubated at $37^{\circ} \mathrm{C}, 5 \%$ CO2 and passaged twice a week using Trypsin-EDTA $(0.25 \%)$ (Life Technologies). Cells were tested for mycoplasma contamination using the MycoAlert Mycoplasma Detection Kit (Lonza), according to the manufacturer's instructions.

Tetraploid formation in human cells. Cells were treated with 0.75 $\mu \mathrm{M}$ actin depolymerizing drug dihydrocytochalasin D (DCD, Sigma) for 18 hours. Subsequently, the drug was washed out $3 x$ using prewarmed PBS. Cells were further cultured in drug-free medium for indicated time or immediately harvested for further experiments.

\section{Construction of post-tetraploids (WGD survivors).}

HCT116 H2B-GFP were treated with $0.75 \mu \mathrm{M}$ of the actomyosin inhibitor dihydrocytochalasin D (DCD, Sigma) for 18 hours. The cells were then washed, placed into a drug-free medium and subcloned by limiting dilution in 96 -well plates ( 0.5 cell per well). After clone expansion, cells were harvested for flow cytometry to measure the DNA content. Subsequently, the genome was analyzed by SNP array analysis and multicolor fluorescent in situ hybridization to validate the tetraploidy. For further details see (17).

RNA isolation. 25 OD600 of yeast cells were collected from YPD exponential cultures, cell pellets were washed twice with ddH2O, then flash frozen in liquid nitrogen. $1 \mathrm{ml}$ of trizol was added to frozen cell pellet and left on ice, cells then were resuspended and transferred to RNase-free screw cap Eppendorf containing $200 \mu \mathrm{l}$ of acid washed nuclease free glass beads. Cells were disrupted by bead beating the tubes using the FastPrep-24TM 5G, 3 cycles of 20 secs at speed $6 \mathrm{~m} / \mathrm{s}$ and 2 minutes in between each cycle. Add $200 \mu \mathrm{l}$ chloroform, vortex $15 \mathrm{sec}$, then incubate at room temp for $5 \mathrm{~min}$. Spin in Epp centrifuge, full speed, $5 \mathrm{~min}, 4^{\circ} \mathrm{C}$. Recover supernatant to a fresh tube, and repeat trizol/ chloroform extraction with $1 \mathrm{ml}$ trizol and $400 \mu \mathrm{l}$ chloroform. Recover supernatant to a fresh tube and precipitate RNA by adding $0.5 \mathrm{ml}$ of isopropanol and standing on ice for 15 minutes. Pellet by cold spin, wash twice with $1 \mathrm{ml} 70 \% \mathrm{EtOH}$, Air dry for 10-20 min, then dissolve in an appropriate amount of RNase-free water depending on the pellet size. Measure the concentration and check the quality by Nano Drop, and agarose gel electrophoresis.

Total RNA was isolated from human cells ( $1 \times 10^{6}$ cells) using the TRIZOL reagent according to the manufacturer's instructions (ThermoFisher Scientific). RNA integrity for each sample was confirmed with by Nano Drop, and agarose gel electrophoresis.

RT-qPCR. To assess the mRNA levels, total mRNA was isolated using a Qiagen mRNeasy mini kit according to manufacturer's protocol. Next, reverse transcription using Anchored-oligo(dT) and Roche Transcriptor First Strand cDNA synthesis Kit (Cat no. 04
379012 001) was performed to obtain cDNA. Quantitative PCR was performed using specific primers and SsoAdvanced Universal SYBR Green Supermix (Bio-Rad, USA). Melting curve analysis was performed to confirm the specificity of amplified product. Each sample was spiked with TATAA Universal RNA Spike II control (TATAA Biocenter AB, Sweden). mRNA expression of each sample was normalized to control housekeeping gene RPL30. The list of used primers is in Supplementary Table 3.

Fluorescence microscopy. Exponentially growing yeast cells were imaged without fixation.

Human cells were seeded and treated when required in a glass-bottom 96-black well plate. The cells were then fixed using ice cold methanol, permeabilized with 3\% Triton X 100 in PBS and blocked in blocking solution (5\% Fetal Bovine Serum + 0.5\% Triton X $100+1 \%$ Na3N in PBS). The DNA was stained DAPI or Vybrant DyeCycle ${ }^{\mathrm{TM}}$ Green for 1 hour in RT. Before imaging, cells were washed 4x with PBS. Imaging was carried out on a spinning disc system comprising of inverted Zeiss Observer.Z1 microscope, Plan Apochromat 63x magnification oil objective, 40x magnification air objective or 20x magnification air objective, epifluorescence X-Cite 120 Series lamp and lasers: 473, 561 and $660 \mathrm{~nm}$ (LaserStack, Intelligent Imaging Innovations, Inc., Göttingen, Germany), spinning disc head (Yokogawa, Hersching, Germany), CoolSNAP-HQ2 and CoolSNAP-EZ CCD cameras (Photometrics, Intelligent Imaging Innovations, Inc., Göttingen, Germany).

Flow cytometry. For DNA content, 1 OD600 of yeast cells growing exponentially in YPD was collected and washed with ddH2O. Cells were fixed in $1 \mathrm{~mL} 70 \%(\mathrm{v} / \mathrm{v})$ ethanol rotating at $4^{\circ} \mathrm{C}$ overnight. Fixed cells were then pelleted at $5000 \mathrm{rpm}$ for 2 minutes and washed with $\mathrm{ddH} 2 \mathrm{O}$. Cells were subsequently resuspended in $500 \mu \mathrm{L} \mathrm{FxCycle}{ }^{\mathrm{TM}}$ PI/RNase staining solution (Life Technologies, F10797), incubated at room temperature in the dark for 30 minutes and then stored at $4^{\circ} \mathrm{C}$ for $72 \mathrm{~h}$ or processed for next step. Samples were sonicated at $40 \%$ amplitude for 15 secs and run on an Attune ${ }^{\mathrm{TM}}$ Flow Cytometer. Data analyses was performed using the FlowJo ${ }^{\mathrm{TM}}$ software, version 10. Human cells were trypsinized and incubated in cold PBS supplemented with 5\% fetal calf serum (Sigma-Aldrich; PBS-FACS). DNA was stained either by propidium iodide (PI); the cells were fixed in cold $70 \%$ ethanol added dropwise while vortexing, and incubated on ice for 30 minutes. Cells were centrifuged and pellets were washed twice with PBS-FACS. $50 \mu \mathrm{l}$ Rase A solution $(100 \mu \mathrm{g} / \mathrm{ml}$ in PBS) was added to the pellet, followed by staining with $400 \mu \mathrm{l}$ PI solution (50 $\mu \mathrm{g} / \mathrm{ml}$ in PBS) per million cells. Cells were incubated for 10' at $25^{\circ} \mathrm{C}$. For Hoechst staining, pellets were incubated in the dark with $10 \mathrm{mg} / \mathrm{ml}$ Hoechst 33358 for $15^{\prime}$ at $4^{\circ} \mathrm{C}$. Data acquisition was performed using the ATTUNE NxT flow cytometer (ThermoFisher). Data analysis was performed using the FlowJo software. Gating strategy: An SSC-A/FSC-A gate was set in order to exclude cell debris, and an FSC-A/FSC-H gate was then set in order to exclude doublets. 
Cell size measurement. Cell volume of budding yeast was determined from microscopy bright field images of exponentially growing cultures using BudJ plugin according to (Ferrezuelo et al., 2012) To determine the cell volume of human cells, exponentially growing cells were harvested and washed with PBS twice. Forward scatter (FSC) was measured by ATTUNE NxT Flow Cytometer (ThermoFisher) and used as the indicator of cell size. Three biological replicated were examined and plotted.

Cell growth for mass spectrometry. The cells were grown in SILAC synthetic drop out medium exponentially at a room temperature. The cell amount in each culture with different ploidy was counted using Burker chamber and equivalent amounts of cells of each ploidy were harvested and washed with PBS. To prepare the SuperSilac, the cells were grown in SILAC heavy medium, counted and mixed 1:1:1:1.

Yeast sample preparation for proteomic analysis. Sample preparation was done as described as in (23). Briefly, cells were lysed in SDS lysis buffer (5\% SDS, $100 \mathrm{mM}$ dithiothreithol, $100 \mathrm{mM}$ Tris $\mathrm{pH}$ 7.6), boiled for $5 \mathrm{~min}$ at $95^{\circ} \mathrm{C}$ and sonicated for $15 \mathrm{~min}$ (Bioruptor Sonicator, $20 \mathrm{kHz}, 320 \mathrm{~W}, 60 \mathrm{~s}$ cycles). Insoluble remnants were removed by centrifugation at $16,000 \mathrm{~g}$ for $5 \mathrm{~min}$ and $140 \mu \mathrm{g}$ clarified protein extract was transferred to a $30 \mathrm{kDa} \mathrm{MW}$ cut-off spin filter (Amicon Ultra $0.5 \mathrm{~mL}$ Filter, Millipore). SDS was completely replaced by repeated washing with $8 \mathrm{M}$ urea. Cysteines were then alkylated using excess amounts of iodoacetamide. Proteins were then proteolytically digested overnight using LysC endoprotease (1:50 $\mathrm{w} / \mathrm{w}$ enzyme to protein). Peptides were eluted and desalted using $\mathrm{C}_{18}$ StageTips.

Liquid chromatography coupled mass spectrometry. MS-based proteomic measurements were performed as in (23). Briefly, approximately $2 \mu \mathrm{g}$ of desalted peptides were loaded and analyzed by linear $4 \mathrm{~h}$ gradients. The LC system was equipped with an in-house made 50-cm, 75- $\mu \mathrm{m}$ inner diameter column slurry-packed into the tip with

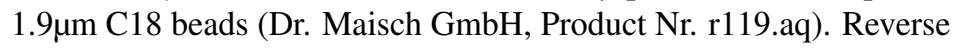
phase chromatography was performed at $50^{\circ} \mathrm{C}$ with an EASY-nLC 1000 ultra-high-pressure system (Thermo Fisher Scientific) coupled to the Q Exactive mass spectrometer (Thermo Fisher Scientific) via a nano-electrospray source (Thermo Fisher Scientific). Peptides were separated by a linear gradient of buffer B up to $40 \%$ in $240 \mathrm{~min}$ for a 4-h gradient run with a flow rate of $250 \mathrm{nl} / \mathrm{min}$. The Q Exactive was operated in the data-dependent mode with survey scans (MS resolution: 50,000 at $\mathrm{m} / \mathrm{z} 400$ ) followed by up to the top $10 \mathrm{MS} 2$ method selecting 2 charges from the survey scan with an isolation window of $1.6 \mathrm{Th}$ and fragmented by higher energy collisional dissociation with normalized collision energies of 25 . Repeated sequencing was avoided using a dynamic exclusion list of the sequenced precursor masses for $40 \mathrm{~s}$.

MS-Data Analysis. Raw files were analyzed by MaxQuant software version 1.6.3.3 (39) and searched against the S. cerevisiae
Uniprot FASTA database (UniProt ID: UP000002311). Lysine0 (light) and Lysine-8 (heavy) were used as SILAC labels. Cysteine carbamidomethylation was set as a fixed modification and $\mathrm{N}$ terminal acetylation and methionine oxidation as variable modifications. LysC/P was set as protease and a maximum of two missed cleavages was accepted. False discovery rate (FDR) was set to 0.01 for peptides (minimum length of 7 amino acids) and proteins and was determined by searching against a generated reverse database. Peptide identification was performed with an allowed initial precursor mass deviation up to $7 \mathrm{ppm}$ and an allowed fragment mass deviation of $20 \mathrm{ppm}$.

Analysis of proteome data. Identified protein groups were filtered to remove contaminants, reverse hits and proteins identified by site only. SILAC light/heavy ratios were calculated and transformed to $\log 2$ scale. Next, Protein groups which were identified more than two times in at least one group of replicates were kept for further processing, resulting in a set of 3109 protein groups in total. To determine significance two-sample T-tests of $2 \mathrm{~N}, 3 \mathrm{~N}$ and $4 \mathrm{~N}$ to $1 \mathrm{~N}$ were performed ( $\mathrm{S} 0=0$, permutation based Benjamini-Hochberg FDR threshold $=0.05$ ). A "combined score" was calculated as the product of the q-values of all two sample tests. The median intensity of the replicates was calculated. The resulting data with statistics can be found in Supplementary Data 1.

Additional annotation (GOBP, GOCC) was added and 2D annotation enrichment analysis performed to identify significantly deregulated pathways (39). YeastEnrichR was used to perform gene set enrichment analysis of statistically significantly different values of all ploidies to haploid. The data is sorted based on the enrichR - combined score, which is a product of the p-value resulting from the Fisher exact test and the z-score of the deviation from the expected rank. Outliers were calculated twice based on the (SILAC L/H) ratio of ratios of each individual ploidy to $1 \mathrm{~N}$. First, the ratios of $4 \mathrm{~N}$ to $1 \mathrm{~N}$ were filtered for the outliers that show the overall strongest up or downregulation. Second, smoothed filtering was performed which additionally excluded values that show differences between consecutive ploidies with a FC $>1$ to remove values which spike between individual ploidies and keep only those that show a consistent trend across ploidies.

PloiDEx - Supplementary plotting application. To allow users the plotting of our combined datasets PloiDEx (Ploidy-Dependent Expression), a suave.io based application has been written in the functional programming language $F$ (4.5.2). The console application has been built in Visual Studio 2019 for the .NET Framework 4.6.1. It allows interactive plotting of both the proteome and transcriptome data, which have been normalized as described previously, in the user's default browser using the graphing library plotly.js in the form of heatmaps and profile plots. For comparability the transcriptome has been matched to the proteome dataset. Filtering for outliers by a threshold and smoothing as described previously have been implemented. Additionally, the application allows the filtering of the dataset by GO cellular compartment or biological processes to plot the associated values from the database. For further information 
about the functionality of the application, used packages and libraries a Readme.pdf has been written, which together with the release and dependent packages is available at:

https://seafile.rlp.net/d/083c634e50d94e10834d/

Dynamic transcriptome analysis (DTA). We used the isogenic strains of different ploidies that were transformed with plasmid YEpEBI311 (2 $\mu \mathrm{m}, L E U 2)$ carrying the human equilibrate nucleoside transporter hENT1. cDTA was performed as previously described (40). S. cerevisiae cells were grown in SD medium overnight, diluted to an $\mathrm{OD}_{600}$ of 0.1 the next day and grown up to a mid-log phase $\left(\mathrm{OD}_{600}\right.$ of 0.8$)$ and labeled with 4-thiouracil (4-tU, Sigma, 2M in DMSO) for $6 \mathrm{~min}$ at a final concentration of $5 \mathrm{mM}$. Schizosaccharomyces pombe cells were grown in YES medium $(5 \mathrm{~g} / \mathrm{liter}$ yeast extract; $30 \mathrm{~g} /$ liter glucose; supplements: $225 \mathrm{mg} /$ liter adenine, histidine, leucine, uracil, and lysine hydrochloride) and labeled with $4 \mathrm{sU}$ $\left(50 \mathrm{mM}\right.$ in $\mathrm{ddH}_{2} \mathrm{O}$ ) for $6 \mathrm{~min}$ at a final concentration of $0.5 \mathrm{mM}$. A final concentration of $5 \mathrm{mM}$ of $4 \mathrm{tU}$ was used. Cells were harvested via centrifugation and cell pellets re-suspended in RNAlater solution (Ambion/Applied Biosystems). The cell concentration was determined using a Cellometer N10 (Nexus) before flash freezing the cells in liquid nitrogen. Total RNA was extracted with the RiboPure-Yeast Kit (Ambion/Applied Biosystems), following the manufacturer's protocol. Labeled RNA was chemically biotinylated and purified using strepatavidin-coated magnetic beads. Labeling of samples for array analysis was performed using the GeneChip 3IVT labeling assay (Affymetrix) with $100 \mathrm{ng}$ input RNA. Samples were hybridized to GeneChip Yeast Genome 2.0 microarrays following the instructions from the supplier (Affymetrix).

cDTA data analysis. Analysis of cDTA data was carried out as described (40) using R/Bioconductor and the DTA package. Briefly, array probes that cross-hybridized between $S$. pombe and $S$. cerevisiae were removed from the analysis. The labeling bias was performed as described (24) and proportional rescaling of the $S$. cerevisiae microarray intensities to the internal $S$. pombe standard was performed to obtain global expression fold changes (40). Differential gene expression analysis was performed using the R Bioconductor package "Limma" and multiple testing correction was done using false discovery rates.

Protein isolation from budding yeast and human cells. Exponentially growing yeast cells were harvested, resuspended in 100 $\mu \mathrm{l}$ lysis buffer and incubated $10 \mathrm{~min}$ on ice. $40 \mu \mathrm{l} 100 \%$ TCA was added and incubated for $10 \mathrm{~min}$ on ice. Precipitated proteins were spun down for $10 \mathrm{~min}$ at $4^{\circ} \mathrm{C}, 13000$. The pellet was washed with $1 \mathrm{ml}$ ice-cold acetone, dried at $50^{\circ} \mathrm{C}$ for 5-20 min and resuspended in $50 \mu \mathrm{l} 2 \mathrm{x}$ Laemmli buffer. Protein lysates were boiled for $5 \mathrm{~min}$ at $96^{\circ} \mathrm{C}$. Pelleted human cells were lysed in RIPA buffer with protease inhibitor cocktail (Pefabloc SC, Roth, Karlsruhe, Germany), then sonicated by ultrasound in a water bath for $15 \mathrm{~min}$. Cell lysate was spun down at $13600 \mathrm{rpm}$ for 10 minutes at $4^{\circ} \mathrm{C}$ on a table-top microcentrifuge (Eppendorf, Hamburg, Germany). $1 \mu \mathrm{l}$ was used to determine protein concentration using Bradford dye at $595 \mathrm{~nm}$ wavelength. Subsequently, the lysates were mixed with 4x Lämmli buffer with $2.5 \%$ ß-mercaptoethanol and boiled at $95^{\circ} \mathrm{C}$ for $5 \mathrm{~min}$.

Immunoblotting. Prepped cell lysates were separated by SDSPAGE using $10 \%$ or $12.5 \%$ gels. Protein size was estimated using the PrecisionPlus All Blue protein marker (Bio-Rad, USA). Gels were incubated in Bjerrum Schafer-Nielsen transfer buffer and proteins were transferred to a water-activated nitrocellulose membranes (Amersham Protran Premium 0.45 NC, GE Healthcare Life Sciences, Sunnyvale, USA) using semi-dry transfer (Trans-Blot ${ }^{\circledR}$ Turbo ${ }^{\mathrm{TM}}$, Bio- Rad, USA). Membranes were stained in Ponceau solution for 5 min and scanned to be used as a loading control. Next, membranes were blocked in 5\% - 10\% skim milk in TBS-T (Fluka, Taufkirchen, Germany) for 1 hour in RT. After blocking, membranes were incubated in respective primary antibodies diluted in $1 \%$ Bovine Serum Albumin (BSA) or $5 \%$ skim milk overnight at $4{ }^{\circ} \mathrm{C}$ with gentle agitation. Further, the membranes were rinsed 3 x 5 minutes with TBS-T, incubated 1 hour in RT with HRP-conjugated secondary antibodies (RD Systems), and followed by rinsing 3x 5 minutes with TBS-T. Chemiluminescence was detected using ECLplus kit (GE Healthcare, Amersham $^{\mathrm{TM}}$ ) and Azure c500 system (Azure Biosystems, Dublin, USA). Protein band quantification was carried out using ImageJ (National Institutes of Health, http://rsb.info.nih.gov/ij/). List of used antibodies is in Supplementary Table 2.

Phos-tagTM SDS-PAGE. Phospho-affinity gel electrophoresis for mobility shift detection of phosphorylated proteins was performed using Phos-tag acrylamide $4.5 \%$ (w/v) running gels polymerized with $25 \mu \mathrm{M}$ Phos-tag acrylamide (FUJIFILM Wako Pure Chemical Corporation) and $50 \mu \mathrm{M} \mathrm{MnCl2}$. Gel running and transfer conditions were optimized according to the manufacturer's protocol.

Puromycin incorporation. Yeast cells were grown exponentially in YPD media then puromycin was added to the culture at a final concentration of $10 \mu \mathrm{M}$ for 15 minutes shaking at $30^{\circ} \mathrm{C}$, then $5 \mathrm{OD}_{600}$ of cells were collected, washed twice with $\mathrm{ddH}_{2} \mathrm{O}$ and prepared for western blot.

Data availability and accession. Transcriptome data have been deposited in the Gene Expression Omnibus database under accession code GSE162513.

The mass spectrometry proteomics data have been deposited to the ProteomeXchange Consortium via the PRIDE partner repository with the dataset identifier PXD022605.

\section{Supplementary Information is available for this paper: \\ Supplementary Figures 1-9 \\ Supplementary Tables 1-4 \\ Supplementary Data Files 1- 3}

